\title{
Gc-Globulin (Vitamin D-binding Protein) Enhances the Neutrophil Chemotactic Activity of C5a and C5a des Arg
}

\author{
Richard R. Kew and Robert O. Webster \\ Departments of Internal Medicine and Microbiology, St. Louis University School of Medicine, St. Louis, Missouri 63104
}

\begin{abstract}
Several serum proteins have been shown to be important in modulating leukocyte chemotaxis and inflammation. We investigated the possibility that the multifunctional serum protein Gc-globulin (vitamin D-binding protein) may also enhance the neutrophil chemotactic activity of complement-derived peptides. Purified Gc-globulin by itself did not induce chemotaxis of human neutrophils. However, as little as $0.01 \mathrm{nM}$ Gc-globulin greatly enhanced the neutrophil chemotactic activity of C5a and its derivative, C5a des Arg over a wide concentration range. The effect was most pronounced at nonchemotactic doses of C5a (0.01 nM) and C5a des Arg (1 nM). Gcglobulin was unable to augment the neutrophil chemotactic activity of FMLP and leukotriene $B_{4}$. This enhancing activity was not due to a nonspecific effect of anionic proteins since other purified serum proteins, of similar size and charge as Gc-globulin ( $\alpha_{1}$ acid glycoprotein, $\alpha_{2}$ HS glycoprotein, $\alpha_{2}$ histidine-rich glycoprotein), could not increase the chemotactic activity of C5a des Arg. Serum depleted of Gc-globulin by immunoaffinity chromatography totally lacked chemotactic enhancing activity for C5a des Arg. Gc-globulin-depleted serum activated with zymosan also had significantly less chemotactic activity than control- (sham-depleted) activated serum. Finally, radioiodinated C5a or C5a des Arg formed a 1:1 complex with purified Gc-globulin when analyzed by gel filtration chromatography. These results indicate that Gcglobulin is the major chemotactic enhancing factor in serum and may function as an up-regulator of the chemotactic activity of C5-derived peptides.
\end{abstract}

\section{Introduction}

Neutrophils are the predominant inflammatory cell type recruited rapidly (within 3-6 h) to sites of tissue injury and/or

Address all correspondence to Dr. Richard Kew, Pulmonary Division, St. Louis University Medical Center, 1325 South Grand Blvd., St. Louis, MO 63104.

Portions of this work were presented at the annual Federation of American Societies of Experimental Biology meetings in Washington, DC, 30 March 1987. This work was published in abstract form in 1987. Fed. Proc. 46(3):445. (Abstr.)

Received for publication 10 August 1987 and in revised form 2 February 1988.

J. Clin. Invest.

(c) The American Society for Clinical Investigation, Inc.

$0021-9738 / 88 / 07 / 0364 / 06 \$ 2.00$

Volume 82, July 1988, 364-369 infection (1). These leukocytes are thought to accumulate at inflammatory sites, largely as a result of local production of chemotactic factors. Although numerous neutrophil chemoattractants have been characterized, less is known about the serum proteins that may regulate their chemotactic activity.

The complement activation peptide C5a $\left(M_{\mathrm{r}} 11,000\right)$ is one of the most potent leukocyte chemoattractants yet described (2). As little as $0.1 \mathrm{nM}$ of purified C5a will induce significant neutrophil chemotaxis in vitro. In the circulation, C5a is rapidly converted to C5a des Arg by the action of serum carboxypeptidase $\mathbf{N}$ (anaphylatoxin inactivator), which removes the $\mathrm{COOH}$-terminal arginine residue (3). The des Arg form of C5a is $\sim 100$-fold less active in provoking neutrophil chemotaxis when tested in vitro (4). However, when assayed in the presence of serum the potency of C5a des Arg is nearly equivalent to that of $\mathrm{C} 5 \mathrm{a}(4,5)$. This observation led investigators to speculate that serum contains a factor(s) that enhances the neutrophil chemotactic activity of C5a des $\operatorname{Arg}(4,5)$. Indeed, Perez et al. subsequently isolated an anionic, $60-\mathrm{kD}$ serum protein, termed cochemotaxin that increased the neutrophil chemotactic activity of C5a des Arg (6). Cochemotaxin is probably the anionic $65-70-\mathrm{kD}, \mathrm{C5}$-associated, chemotactic factor reported by others $(7,8)$.

We hypothesized that Gc-globulin (vitamin D-binding protein) may be this serum chemotactic enhancing factor, since it possesses many of the same biochemical properties (such as molecular weight [6-8], isoelectric point [7], heat stability [6, 7], and sensitivity to disulfide reducing agents [7]) that have been previously described for cochemotaxin and/or the 65-70-kD anionic serum factor. Gc-globulin is an anionic protein (pI 4.95-5.1 depending on the isotype) with an $M_{\mathrm{r}}$ of $\sim 52,000$ and a serum concentration of $0.3-0.4 \mathrm{mg} / \mathrm{ml}(9,10)$. Besides acting as the serum transport protein for vitamin $D$, Gc-globulin is also associated with membrane immunoglobulin of B lymphocytes $(11,12)$ and a subset of $\mathrm{T}$ lymphocytes (13). Gc-globulin is also an actin-binding protein and can form a tight 1:1 molar complex with G-actin monomers (14) and recently, it has been demonstrated that Gc-globulin-actin complexes are rapidly cleared from the circulation by the liver (15). In this report, we demonstrate that Gc-globulin can also enhance the neutrophil chemotactic activity of the complement peptides C5a and C5a des Arg.

\section{Methods}

Purified proteins and peptides. C5a was isolated from zymosan-activated human serum according to the method of Fernandez and Hugli (2) with slight modifications suggested by Hugli et al. (16). Briefly, a 
pool of normal human serum (NHS) ${ }^{1}$ was first treated with the carboxypeptidase $\mathrm{N}$ inhibitor DL-2-mercaptomethyl-3-guanidino-ethylthiopropanoic acid at a concentration of $1 \mathrm{mM}$ (Calbiochem-Behring Corp., La Jolla, CA). Next, serum was activated with boiled zymosan particles $(20 \mathrm{mg} / \mathrm{ml})$ for $45 \mathrm{~min}$ at $37^{\circ} \mathrm{C}$. After acid precipitation ( 1 $\mathrm{N} \mathrm{HCl}$ ), the activated serum supernate was subjected to gel filtration chromatography on Bio-Gel P-60 (Bio-Rad Laboratories, Richmond, CA) equilibrated with $0.1 \mathrm{M}$ ammonium formate (pH 7.0). Finally, C5a-containing fractions were further purified using fast protein liquid chromatography with a Mono S cation exchange column (Pharmacia Fine Chemicals, Piscataway, NJ). C5a des Arg was generated by treating purified C5a with solid phase carboxypeptidase B (17). Highly purified Gc-globulin (mixed isotypes), as well as $\alpha_{2}$ HS glycoprotein, $\alpha_{1}$ acid glycoprotein, and $\alpha_{2}$ histidine-rich glycoprotein were purchased from Calbiochem-Behring Corp. We determined these proteins to be $>99 \%$ pure by SDS-PAGE and immunoelectrophoresis vs anti-whole human serum.

Neutrophil chemotaxis assay. Neutrophils were obtained by standard procedures (18), from the blood of healthy, medication-free volunteers who gave informed consent. Chemotaxis was quantitated using two-compartment, blind-well chemotaxis chambers and cellulose nitrate filters (3.0- $\mu \mathrm{m}$ pore size, 13-mm diameter; Sartorius Filters, Inc., Hayward, CA), which separate the upper from lower compartments. All samples and cell suspensions were prepared or diluted in HBSS with $10 \mathrm{mM}$ Hepes (pH 7.4) and 2\% BSA. Samples and buffer controls were added in duplicate to the lower wells of the chemotaxis chamber and $0.2 \mathrm{ml}$ of a neutrophil suspension $\left(3.75 \times 10^{6}\right.$ neutrophils $\left./ \mathrm{ml}\right)$ was then added to the upper wells. After a $45-\mathrm{min}$ incubation at $37^{\circ} \mathrm{C}$, filters were removed, fixed in $100 \%$ 2-propanol, stained with hematoxylin, and mounted on microscope slides. Neutrophil movement into the filter was quantitated using the leading front method (19). Five $400 \times$ fields were measured for each filter and all samples were assayed in duplicate. The mean value of the buffer control (random neutrophil movement) was subtracted from each sample so that the data are reported as net migration distance (micrometers/45 min).

Preparation of Gc-globulin-depleted serum. Gc-globulin-depleted serum was prepared using a pool of NHS that was obtained from the blood of six healthy, nonsmoking, medication-free volunteers who gave informed consent. The polyclonal IgG fraction of goat antihuman Gc-globulin was purchased from Atlantic Antibodies (Scarborough, ME). Anti-Gc-globulin was covalently linked to Sepharose 4B (Pharmacia Fine Chemicals) according to the method of March et al. (20). Heat-inactivated (at $56^{\circ} \mathrm{C}$ for $30 \mathrm{~min}$ ) NHS was incubated with anti-Gc-globulin-Sepharose at $22^{\circ} \mathrm{C}$ at a ratio of packed gel to NHS of 5:1 (vol/vol). After being mixed constantly for $45 \mathrm{~min}$, serum was separated from the Sepharose beads by centrifugation (at $1500 \mathrm{~g}$ for $15 \mathrm{~min}$ ). To minimize nonspecific adsorption of proteins to the Sepharose and/or immunoglobulin, the reaction was performed in the presence of $0.5 \mathrm{M} \mathrm{NaCl}$. To confirm that only $\mathrm{Gc}$-globulin was removed from serum by this procedure, the anti-Gc-globulin-Sepharose was first washed 5 times in $0.5 \mathrm{M} \mathrm{NaCl}$ and $20 \mathrm{mM} \mathrm{NaPO}_{4}$ (pH 7.5). Then protein was eluted from the antibody using $0.1 \mathrm{M}$ glycine $(\mathrm{pH}$ 2.3). Only one protein, $M_{\mathrm{r}} 52,000$ and immunologically reactive with anti-Gc-globulin, was identified by SDS-PAGE and immunoelectrophoresis against anti-whole human serum. An aliquot of NHS was reacted with Sepharose $4 B$ under identical conditions and this shamdepleted material was designated as control serum. Both control and Gc-globulin depleted serum, diluted to $1 \%$ in chemotaxis assay buffer, were examined for neutrophil chemotactic activity in the presence or absence of $1 \mathrm{nM}$ C5a des Arg.

Zymosan activation of Gc-globulin-depleted serum. For the zymosan activation experiments, NHS was depleted of Gc-globulin or sham depleted as above except that the starting serum pool was not heat inactivated. Depleted serum was also concentrated approximately five-

1. Abbreviations used in this paper: NHS, normal human serum. fold, using a Centricon 10 microconcentrator (10,000-D filtration limit; Amicon Corp., Danvers, MA), to match the absorbance value at $280 \mathrm{~nm}$ of the starting serum pool. (We found this step to be critical since the depletion procedure dilutes serum to such an extent that the alternative pathway of complement can no longer be activated by zymosan.) After addition of $\mathrm{CaCl}_{2}$ and $\mathrm{MgCl}_{2}$ to a final concentration of $1 \mathrm{mM}, 1 \mathrm{ml}$ of depleted serum was activated with $10 \mathrm{mg}$ of washed zymosan particles for $45 \mathrm{~min}$ at $37^{\circ} \mathrm{C}$ with constant mixing. Zymosan particles were then removed by centrifugation using a microfuge $\left(15,000 \mathrm{~g}\right.$ for $2 \mathrm{~min}$ at $\left.4^{\circ} \mathrm{C}\right)$ and the zymosan-activated, depleted serum was then examined for neutrophil chemotactic activity as described above.

Gel filtration chromatography of radioiodinated C5a-Gc-globulin complexes. $\mathrm{C} 5 \mathrm{a}$ and $\mathrm{C5}$ a des Arg $(1 \mu \mathrm{g})$ were radioiodinated using a solid phase lactoperoxidase-glucose oxidase system (Enzymobeads; Bio-Rad Laboratories) and ${ }^{125} \mathrm{I}-\mathrm{Na}$ (New England Nuclear, Boston, MA) according to the manufacturer's instructions. Radioiodinated C5a (and des Arg) was then separated from both aggregated peptides and free ${ }^{125}$ I-Na by gel filtration on Sephacryl S-200 (Pharmacia Fine Chemicals). Specific activity of the C5-derived peptides was $1 \mu \mathrm{Ci} / \mu \mathrm{g}$ with $>90 \%$ of the activity precipitable with TCA. Approximately 50 $\mathrm{ng}{ }^{125} \mathrm{I}-\mathrm{C} 5 \mathrm{a}( \pm 50 \mu \mathrm{g} \mathrm{Gc}$-globulin) in a total of $0.2 \mathrm{ml}$ was applied to a 1 $\times 30 \mathrm{~cm}$ column of Bio-Gel P-100 (Bio-Rad Laboratories) equilibrated with PBS containing $1 \mathrm{mM} \mathrm{Ca}^{2+}$ and $\mathrm{Mg}^{2+}$. $0.5-\mathrm{ml}$ fractions were then collected at a flow rate of $6 \mathrm{ml} / \mathrm{h}$ and radioactivity was quantitated using a gamma counter.

Statistical analysis. Data were analyzed for statistical significance using either $t$ test (comparing two samples) or the T method for unplanned comparisons among means (comparing three or more samples) (21).

\section{Results}

Purified Gc-globulin enhanced the neutrophil chemotactic activity of C5a and C5a des Arg in a concentration-dependent manner (Fig. 1). $100 \mathrm{nM} \mathrm{Gc-globulin}$ enhanced the chemotactic activity at almost all concentrations of the peptides

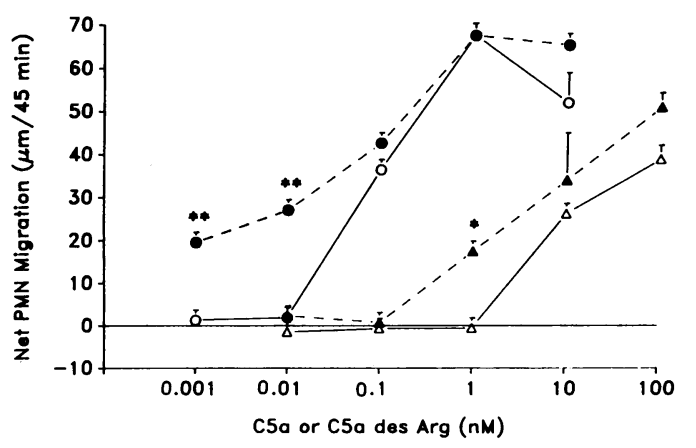

Figure 1. Enhancement of the neutrophil chemotactic activity of C5a and C5a des Arg by Gc-globulin. Purified human C5a or C5a des Arg, $\pm 100 \mathrm{nM} \mathrm{Gc}$-globulin, was incubated at $22^{\circ} \mathrm{C}$ for $30 \mathrm{~min}$ in HBSS with $10 \mathrm{mM}$ Hepes, pH 7.4, and $2 \%$ BSA. Samples and buffer controls were then examined for chemotactic activity as described in Methods. The mean value of the buffer control (random neutrophil movement) was subtracted from each sample so that the data are reported as net migration distance (micrometers/45 min). Asterisks indicate significantly greater $\left({ }^{* *} P<0.001,{ }^{*} P<0.01\right)$ than corresponding sample without $100 \mathrm{nM} \mathrm{Gc}$-globulin. Data are expressed as mean \pm SEM of three experiments using neutrophils from different donors. - - - - - , C5a + 100 nM Gc-globulin; - o-, C5a; -,--- C5a des Arg + 100 nM Gc-globulin; and $-\Delta-$, C5a des Arg. PMN = neutrophils. 


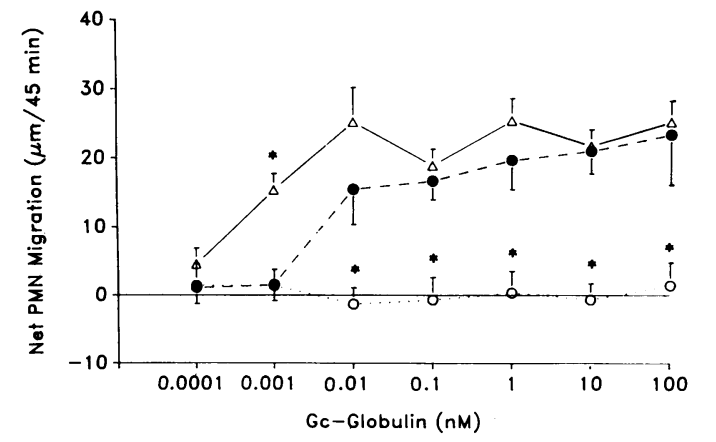

Figure 2. Effect of Gc-globulin concentration on the neutrophil chemotactic activity of C5a and C5a des Arg. Various concentrations of Gc-globulin were tested for chemotactic activity in the presence or absence of $0.01 \mathrm{nM} \mathrm{C5a}$ and $1 \mathrm{nM} \mathrm{C5a}$ des Arg. *Significantly different (either higher or lower at $P<0.01$ ) than all other groups at the indicated concentration of Gc-globulin. Data represent mean \pm SEM of five to seven experiments. $-\Delta-$, Gc-globulin $+0.01 \mathrm{nM} \mathrm{C5a}$; $---\bullet---$, Gc-globulin + 1 nM C5a des Arg; and - - - --- , Gc-globulin. PMN = neutrophils.

tested, but the effect was most pronounced, and statistically significant, at levels of C5a (0.01 nM) and C5a des Arg (1 nM) which, by themselves, were not chemotactic for neutrophils. By varying the concentration of Gc-globulin and C5a or C5a des Arg above and below the filter, it was also determined that this enhancing effect was not due to chemokinesis (random activated movement) but represented true chemotaxis (data not shown). Fig. 2 illustrates the effective concentration range of the chemotactic enhancing activity of Gc-globulin for nonchemotactic amounts of C5a and C5a des Arg. Gc-globulin by itself did not induce neutrophil chemotaxis. However, as little as $0.01 \mathrm{nM} \mathrm{Gc-globulin}$ significantly augmented $(P<0.01)$ the chemotactic activity of $1 \mathrm{nM}$ C5a des Arg, and 1 pM Gc-globulin was capable of provoking neutrophil chemotaxis to 0.01 nM C5a. The chemotactic enhancing activity of Gc-globulin was specific for the complement peptides as demonstrated in Fig. 3, in which it was observed that Gc-globulin does not enhance the neutrophil chemotactic activity of the tripeptide FMLP. A higher concentration of Gc-globulin, $100 \mathrm{nM}$, gave similar negative results with FMLP and was unable to augment the chemotactic activity of another well-characterized neutrophil chemoattractant, leukotriene $\mathrm{B}_{4}\left(\mathrm{LTB}_{4}\right.$, data not

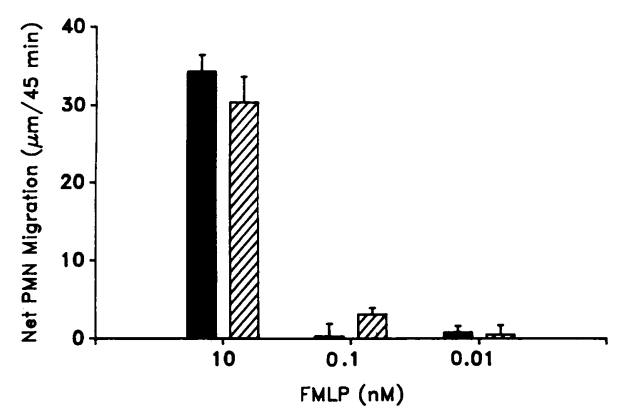

Figure 3. Effect of Gc-globulin on FMLP-induced neutrophil chemotaxis. Various concentrations of FMLP were examined for chemotactic activity in the presence or absence of $10 \mathrm{nM} \mathrm{Gc-globulin.} \mathrm{Data}$ represent mean \pm SEM of four experiments. a, FMLP; 6 , FMLP + 10 nM Gc-globulin. PMN = neutrophils.
Table I. Effect of Purified Anionic Serum Proteins on the Chemotactic Activity of C5a des Arg

\begin{tabular}{lcrrr}
\hline & & \multicolumn{3}{c}{$\begin{array}{c}\text { Protein concentration } \\
\text { (micrograms per milliliter) }\end{array}$} \\
\cline { 3 - 5 } \multicolumn{1}{c}{ Serum protein } & $\begin{array}{c}1 \mathrm{nM} \\
\text { C5a des Arg }\end{array}$ & \multicolumn{1}{c}{50} & \multicolumn{1}{c}{1.0} & 0.05 \\
\hline$\alpha_{2}$ HS glycoprotein & - & $-4 \pm 1.9$ & $-5 \pm 2.3$ & $2 \pm 3.5$ \\
& + & $0 \pm 5.0$ & $-5 \pm 2.7$ & $5 \pm 2.5$ \\
$\alpha_{2}$ Histidine-rich & - & $-4 \pm 3.0$ & $-5 \pm 2.6$ & $-4 \pm 2.3$ \\
glycoprotein & + & $-4 \pm 2.5$ & $-5 \pm 3.5$ & $-4 \pm 3.1$ \\
$\alpha_{1}$ Acid glycoprotein & - & $2 \pm 3.3$ & $6 \pm 6.9$ & $-4 \pm 2.1$ \\
& + & $-1 \pm 3.9$ & $-4 \pm 2.1$ & $-2 \pm 4.3$ \\
Gc-globulin & - & $0 \pm 1.7$ & $0 \pm 0.4$ & $-1 \pm 0.1$ \\
& + & $14 \pm 0.3$ & $13 \pm 0.4$ & $13 \pm 0.8$ \\
& & & & \\
\hline
\end{tabular}

The indicated concentrations of each purified serum protein were examined for their ability to induce neutrophil chemotaxis in the presence or absence of $1 \mathrm{nM}$ C5a des Arg. Data represent mean \pm SEM ( $n$ $=2-3$ ) of net neutrophil migration distance $(\mu \mathrm{m} / 45 \mathrm{~min})$. Negative values indicate that migration was less than that seen with the buffer control.

shown). The specificity of the serum-enhancing activity was demonstrated in that three highly purified anionic serum proteins, whose sizes and charges are similar to that of Gc-globulin, failed to augment thechemotactic activity of $1 \mathrm{nM} \mathrm{C5a}$ des Arg (Table I). Additionally, DNase I, a protein with actinbinding properties similar to those of Gc-globulin, was unable to enhance the chemotactic activity of C5a des Arg (data not shown), indicating that the chemotactic enhancing activity is specific to Gc-globulin and is not peculiar to actin monomer binding proteins. NHS depleted of Gc-globulin (by immunoaffinity chromatography) failed to augment the chemotactic activity of C5a des Arg. Fig. 4 demonstrates that both control and Gc-globulin-depleted serum lacked neutrophil chemotactic activity when tested alone. (1\% serum was chosen because the control serum had no intrinsic chemotactic activity at this dilution.) However, when $1 \mathrm{nM}$ C5a des Arg was added, only

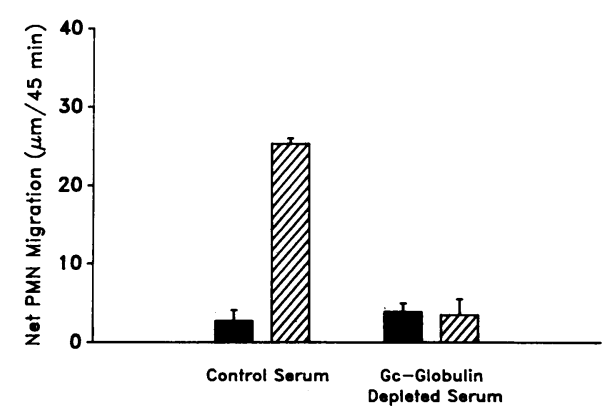

Figure 4. Gc-globulin-depleted serum lacks neutrophil chemotactic enhancing activity. Both control (sham-depleted) and Gc-globulindepleted serum, diluted to $1 \%$ in chemotaxis assay buffer, were examined for neutrophil chemotactic activity in the presence or absence of $1 \mathrm{nM}$ C5a des Arg. Control serum $+1 \mathrm{nM} \mathrm{C5a}$ des Arg is significantly more chemotactic than all other groups $(P<0.001)$. Data represent mean \pm SEM of four experiments using two different preparations of Gc-globulin-depleted serum. a, 1\% serum; ; $1 \%$ serum + 1 nM C5a des Arg. PMN = neutrophils. 
the control serum containing Gc-globulin could significantly augment $(P<0.001)$ chemotactic activity, whereas Gc-globulin-depleted serum completely lacked any demonstrable enhancing activity. Higher concentrations of Gc-globulin-depleted serum (up to $20 \%$ ) also lacked enhancing activity (data not shown). This indicates that Gc-globulin is the major chemotactic enhancing factor present in serum. The neutrophil chemotactic activity of zymosan-activated, Gc-globulin-depleted serum is shown in Fig. 5. (10\% activated serum was examined since this dilution induced maximal neutrophil chemotaxis for both control and Gc-globulin-depleted serum.) Zymosan-activated control serum was significantly $(P$ $<0.001)$ more chemotactic for neutrophils than activated Gcglobulin-depleted serum, indicating that Gc-globulin is required for maximal chemotactic activity in zymosan-activated serum. Comparisons of higher $(20 \%)$ and lower $(5 \%)$ concentrations of zymosan-activated depleted serum also produced similar differences (data not shown). Finally, Fig. 6 demonstrates that C5a forms a 1:1 complex with Gc-globulin when analyzed by gel filtration chromatography. Purified radioiodinated C5a eluted with a molecular mass of $\sim 10-12 \mathrm{kD}$ when chromatographed on Bio-Gel P-100. However, when pure Gc-globulin was mixed with ${ }^{125} \mathrm{I}-\mathrm{C} 5 \mathrm{a}$, two peaks of radioactivity were observed. The first peak eluted with a molecular mass of $\sim 65 \mathrm{kD}$ and the second with a molecular mass of $\sim 11 \mathrm{kD}$. The $65-\mathrm{kD}$ peak indicates that ${ }^{125} \mathrm{I}-\mathrm{C} 5 \mathrm{a}(11 \mathrm{kD})$ formed a 1:1 complex with Gc-globulin (52 kD), and the second peak corresponds to free ${ }^{125} \mathrm{I}-\mathrm{C} 5 \mathrm{a}$. Experiments with radioiodinated C5a des Arg gave identical results (data not shown).

\section{Discussion}

The results indicate that Gc-globulin can function as an upregulator of complement-induced neutrophil chemotaxis. The chemotactic factor enhancing activity of Gc-globulin appears to be specific for C5a or C5a des Arg, since the chemotactic activity of other chemoattractants (FMLP, LTB $_{4}$ ) was unaffected by Gc-globulin. In addition, the chemotactic activity of C5a des Arg could not be augmented by anionic serum pro-

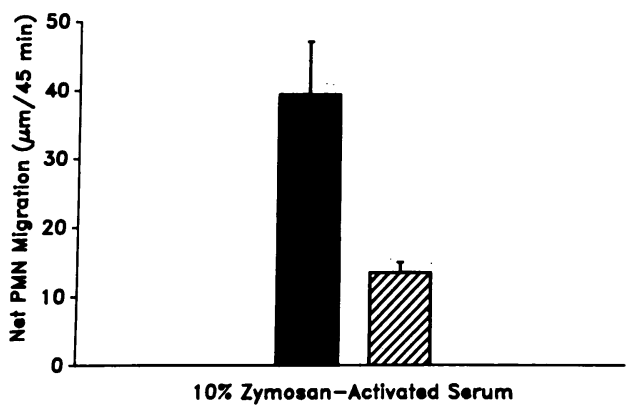

Figure 5. Zymosan-activated, Gc-globulin-depleted serum is less chemotactic than control (sham-depleted) activated serum. Zymosan-activated serum samples were diluted to $10 \%$ in chemotaxis assay buffer and then examined for neutrophil chemotactic activity. Control activated serum is significantly more chemotactic $(P<0.001)$ than $\mathrm{Gc}$-globulin depleted serum. Data represent mean \pm SEM of three experiments. $₫$, control serum; 0 , Gc-globulin-depleted serum. PMN = neutrophils.

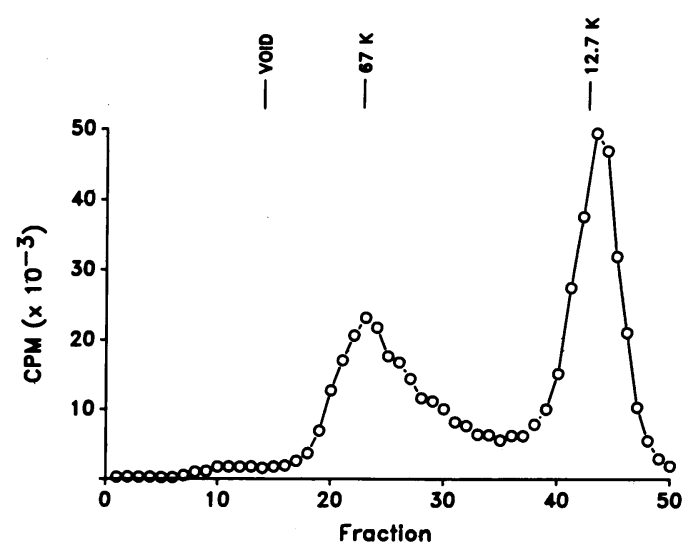

Figure 6. Elution profiles of ${ }^{125} \mathrm{I}-\mathrm{C} 5 \mathrm{a}$ and Gc-globulin on gel filtration chromatography. $50 \mathrm{ng}{ }^{125} \mathrm{I}-\mathrm{C} 5 \mathrm{a}+50 \mu \mathrm{g} \mathrm{Gc}$-globulin were chromatographed on a $1 \times 30 \mathrm{~cm}$ column of Bio-Gel P-100 (Bio-Rad Laboratories) equilibrated with PBS containing $1 \mathrm{mM} \mathrm{CaCl}_{2}$ and 1 $\mathrm{mM} \mathrm{MgCl} 2.0 .5-\mathrm{ml}$ fractions were collected at a flow rate of $6 \mathrm{ml} / \mathrm{h}$. Elution of standard markers is indicated by the arrows: void (blue dextran), $67 \mathrm{~K}$ (BSA), $12.7 \mathrm{~K}$ (cytochrome $c$ ).

teins, similar in size to Gc-globulin, or by another actin-binding protein (DNase I). Perhaps the strongest evidence for Gcglobulin as the major serum chemotactic enhancing factor is provided by the depletion experiments (Figs. 4 and 5), in which Gc-globulin-depleted serum totally lacked chemotactic enhancing activity for C5a des Arg (Fig. 4), and zymosan-activated, Gc-globulin-depleted serum was significantly less chemotactic than activated control serum (Fig. 5). In vivo, Gcglobulin may be an important proinflammatory molecule outside the vasculature, where complement can readily be activated. Gc-globulin has been detected in human bronchoalveolar lavage at concentration of $\sim 0.1 \mu \mathrm{M}(22)$. This amount is several orders of magnitude greater than that needed to augment the chemotactic activity of subchemotactic concentrations of C5a or C5a des Arg (Fig. 2). Furthermore, carboxypeptidase $\mathrm{N}\left(M_{\mathrm{r}} 310,000\right)$ is normally not found outside the circulation; therefore, any complement activation in the tissues would generate primarily, at least transiently, C5a. Moreover, the phlogistic properties of C5a are 100- to 1000-fold greater than C5a des Arg $(3,17)$ and Figs. 1 and 2 demonstrate that the combination of Gc-globulin and C5a is more chemotactic than Gc-globulin plus the des Arg form. Both C5a and C5a des Arg are also known to stimulate other neutrophil functions, such as degranulation and superoxide anion generation (17). However, we have observed that Gc-globulin is unable to enhance these neutrophil functions when stimulated with either complement peptide (Kew, R. R., and R. O. Webster, unpublished observations).

The serum chemotactic enhancing factor termed cochemotaxin may be Gc-globulin. Indeed, we have evidence that indicates that purified cochemotaxin reacts with polyclonal anti-Gc-globulin antibody in a dot-blot assay (Kew, R. R., and R. O. Webster, unpublished observations). However, there are some discrepancies between the enhancing activity previously described for cochemotaxin and that for Gc-globulin reported herein. First, it has been reported that cochemotaxin augments the chemotactic activity of C5a des Arg, but not C5a (6). Enhancing activity for both peptides is supported by the work of 
Beebe et al., who showed that complement-derived chemotactic activity in zymosan-activated serum, in part, corresponded to an anionic $65-\mathrm{kD}$ protein (perhaps C5a or C5a des Arg + Gc-globulin) in the presence or absence of a carboxypeptidase $\mathrm{N}$ inhibitor, indicating that both $\mathrm{C} 5 \mathrm{a}$ or $\mathrm{C} 5 \mathrm{a}$ des Arg were complexed (7). Second, the cochemotaxin-enhanced neutrophil chemotactic activity of C5a des Arg is almost equivalent to that seen with an optimal concentration of C5a, whereas Gc-globulin-enhanced C5a des Arg-induced chemotaxis is considerably less than that of C5a. This latter observation may be explained by differences in the activity between the C5a preparations since our $\mathrm{C} 5 \mathrm{a}$, using a peak chemotactic concentration, was almost twice as potent in provoking neutrophil chemotaxis as the C5a used by Perez et al. (6).

Gc-globulin is clearly distinct from cocytotaxin as described by Wissler et al. (23). Cocytotaxin is a small $\left(M_{\mathrm{r}} 8500\right)$ cationic peptide that appears to restore the neutrophil (but not macrophage) chemotactic activity of inactive $\mathrm{C} 5 \mathrm{a}$, and has only been found in rodent and porcine serum.

It should be emphasized that our investigation examined the effect of Gc-globulin on neutrophil chemotaxis only. The ability of Gc-globulin to enhance complement-induced chemotaxis of other cell types has not yet been determined, although it has been reported that cochemotaxin failed to augment monocyte chemotactic activity of C5a des Arg (24). The mechanism by which Gc-globulin enhances complement-induced neutrophil chemotaxis is not known. Perez et al. have shown that cochemotaxin forms a 1:2 complex with C5a des Arg with all the ${ }^{125} \mathrm{I}-\mathrm{C} 5 \mathrm{a}$ des Arg complexing with cochemotaxin (25). However, we observed that Gc-globulin and C5a (or des Arg) form a 1:1 complex with $\sim 50 \%$ of the ${ }^{125} \mathrm{I}-\mathrm{C} 5 \mathrm{a}$ complexing to Gc-globulin (Fig. 6). Previous studies with the anionic $65-70-\mathrm{kD}, \mathrm{C5}$-associated chemotactic factor from complement-activated serum demonstrated that a portion of $\mathrm{C5a}$ (or C5a des Arg) chemotactic activity was observed at a molecular mass of $65-70 \mathrm{kD}$, indicating that only some of the C5-derived peptides formed a 1:1 complex. The reason for this partial binding is not clear, although one would assume that all the C5a (or des Arg) should be bound to Gc-globulin since in serum it is in great molar excess. This may indicate that only a subset of Gc-globulin isotypes actually bind the C5-derived peptides.

Cell surface Gc-globulin has been reported on several types of leukocytes (12), and therefore may act to bind and concentrate the C5-derived peptides on the neutrophil surface, thereby enhancing the activity of subchemotactic concentrations of the peptides. Since the carbohydrate moiety of C5a des Arg is known to modulate its biological activity (26), Gc-globulin may function by binding the oligosaccharide side chains of the C5-derived peptides as has been proposed for the action of cochemotaxin on C5a des Arg (25). It has been shown that soluble sialic acid can dissociate cochemotaxin- ${ }^{125} \mathrm{I}-\mathrm{C} 5 \mathrm{a}$ des Arg complexes (25). Indeed, we were initially unsuccessful in demonstrating Gc-globulin-C5a complexes by gel filtration fast performance liquid chromatography using an agarose matrix column (Superose 12). After switching to a carbohydratefree polyacrylamide matrix (Bio-Gel P-100) we obtained evidence of complex formation (Fig. 6).

In conclusion, the data presented indicate that $\mathrm{Gc}$-globulin is the major serum-enhancing factor for complement chemotactic peptides and as such is likely to enhance the recruitment of neutrophils to inflammatory foci.

\section{Acknowledgments}

This work was supported by grants HL-30572 and HL-07050 from the U. S. Public Health Service. Dr. Webster is the recipient of a National Institutes of Health Research Career Development Award, HL-01542.

\section{References}

1. Baggiolini, M., and B. Dewald. 1985. The neutrophil. Int. Arch. Allergy Appl. Immunol. 76(Suppl. 1):13-20.

2. Fernandez, H. N., and T. E. Hugli. 1976. Partial characterization of human C5a anaphylatoxin. J. Immunol. 117:1688-1694.

3. Hugli, T. E. 1984. Structure and function of the anaphylatoxins. Springer Semin. Immunopathol. 7:193-219.

4. Fernandez, H. N., P. M. Henson, A. Otani, and T. E. Hugli. 1978. Chemotactic response to human C3a and C5a anaphylatoxins. $J$. Immunol. 120:109-115.

5. Perez, H. D., I. M. Goldstein, D. Chernoff, R. O. Webster, and P. M. Henson. 1980. Chemotactic activity of C5a des Arg: evidence of a requirement for an anionic peptide "helper factor" and inhibition by a cationic protein in serum from patients with systemic lupus erythematosus. Mol. Immunol. 17:163-169.

6. Perez, H. D., I. M. Goldstein, R. O. Webster, and P. M. Henson. 1981. Enhancement of the chemotactic activity of human C5a des Arg by an anionic polypeptide ("cochemotaxin") in normal serum and plasma. J. Immunol. 126:800-804.

7. Beebe, D. P., P. A. Ward, and J. K. Spitznagel. 1980. Isolation and characterization of an acidic chemotactic factor from complement-activated human serum. Clin. Immunol. Immunopathol. 15:88105.

8. Hakansson, L., and P. Venge. 1984. The chemotactic activity of normal and complement-activated serum as measured by the leading front method using a Boyden chamber. Scand. J. Immunol. 19:63-73.

9. Svasti, J., A. Kurosky, A. Bennett, and B. H. Bowman. 1979. Molecular basis for the three major forms of human serum vitamin D binding protein (group-specific component). Biochemistry. 18:16111617.

10. Van Baelen, H., R. Bouillon, and P. De Moor. 1978. The heterogeneity of human Gc-globulin. J. Biol. Chem. 253:6344-6345.

11. Petrini, M., D. L. Emerson, and R. M. Galbraith. 1983. Linkage between surface immunoglobulin and cytoskeleton of $B$ lymphocytes may involve Gc protein. Nature (Lond.). 306:73-74.

12. Petrini, M., R. M. Galbraith, P. A. M. Werner, D. L. Emerson, and P. Arnaud. 1984. Gc (vitamin D binding protein) binds to cytoplasm of all human lymphocytes and is expressed on B-cell membranes. Clin. Immunol. Immunopathol. 31:282-295.

13. Petrini, M., R. M. Galbraith, D. L. Emerson, A. E. Nel, and P. Arnaud. 1985. Structural studies of T lymphocyte Fc receptors. Association of $\mathrm{Gc}$ protein with IgG binding to $\mathrm{Fc}_{r}$. J. Biol. Chem. 260:18041810.

14. Van Baelen, H., R. Bouillon, and P. De Moor. 1980. Vitamin D-binding protein (Gc-globulin) binds actin. J. Biol. Chem. 255:2270-2272.

15. Lind, S. E., D. B. Smith, P. A. Janmey, and T. P. Stossel. 1986. Role of plasma gelsolin and the vitamin D-binding protein in clearing actin from the circulation. J. Clin. Invest. 78:736-742.

16. Hugli, T. E., C. Gerard, M. Kawahara, M. E. Scheetz II, R. Barton, S. Briggs, G. Koppel, and S. Russell. 1981. Isolation of three separate anaphylatoxins from complement-activated human serum. Mol. Cell. Biochem. 41:59-66.

17. Webster, R. O., S. R. Hong, R. B. Johnston, Jr., and P. M. Henson. 1980. Biological effects of the human complement fragments $\mathrm{C} 5 \mathrm{a}$ and C5a des Arg on neutrophil function. Immunopharmacology. 2:201-219.

18. Boyum, A. 1968. Isolation of mononuclear cells and granulo- 
cytes from human peripheral blood. Scand. J. Clin. Lab. Invest. 21(Suppl. 97):77-89.

19. Zigmond, S. H., and J. G. Hirsch. 1973. Leukocyte locomotion and chemotaxis. New methods for evaluation and demonstration of a cell-derived chemotactic factor. J. Exp. Med. 137:387-410.

20. March, S. C., I. Parikh, and P. Cuatrecasas. 1974. A simplified method for cyanogen bromide activation of agarose for affinity chromatography. Anal. Biochem. 60:149-152.

21. Sokal, R. R., and F. J. Rohlf. Biometry. 2nd ed. W. H. Freeman \& Co., Publishers, New York. 222-229, 242-262.

22. Bell, D. Y., J. A. Haseman, A. Spock, G. McLennan, and G. E. R. Hook. 1981. Plasma proteins of the bronchoalveolar surface of the lungs of smokers and nonsmokers. Am. Rev. Respir. Dis. 124:72-79.
23. Wissler, J. H., E. Sorkin, and V. J. Stecher. 1974. Regulation of serum-derived chemotactic activity by the leucotactic binary peptide system. Antibiot. Chemother. (Basel). 19:442-463.

24. Marder, S. R., D. E. Chenoweth, I. M. Goldstein, and H. D. Perez. 1985. Chemotactic responses of human peripheral blood monocytes to the complement-derived peptides C5a and C5a des Arg. $J$. Immunol. 134:3325-3331.

25. Perez, H. D., D. E. Chenoweth, and I. M. Goldstein. 1986. Attachment of human C5a des Arg to its cochemotaxin is required for maximal expression of chemotactic activity. J. Clin. Invest. 78:15891595.

26. Gerard, C., and T. E. Hugli. 1981. Identification of classical anaphylatoxin as the des Arg form of the C5a molecule: Evidence of a modulator role for the oligosaccharide unit in human des $\mathrm{Arg}^{74}-\mathrm{C} 5 \mathrm{a}$. Proc. Natl. Acad. Sci. USA. 78:1833-1837. 